027.7 Zeitschrift für Bibliothekskultur / Journal for Library Culture • Ausgabe 8(2)

\title{
Transformationsverträge - Stairway to Heaven oder Highway to Hell?
}

Bernhard Mittermaier

Published on: Nov 08, 2021

License: Creative Commons Attribution 4.0 International License (CC-BY 4.0). 


\section{ABSTRACT}

Transformationsverträge ermöglichen den lesenden Zugriff auf Zeitschriften und regeln gleichzeitig das Open-Access-Publizieren in ihnen. Mit

Transformationsverträgen verbinden sich Hoffnungen, zum Beispiel auf eine Transformation des Publikationswesens, ebenso wie Befürchtungen, zum Beispiel dass andere Verlage durch Transformationsverträge ungebührlich benachteiligt werden. Zur Untersuchung solcher Fragestellungen wurde der Publikationsoutput von Deutschland, Österreich und der Schweiz untersucht. Im Ergebnis werden keine negativen Auswirkungen durch Transformationsverträge festgestellt, allerdings erfüllen sich bislang auch die Hoffnungen auf substantielle Transformationsschritte nicht.

Transformative agreements enable reading access to journals and at the same time regulate open access publishing in them. Transformation agreements are associated with hopes, e.g. for a transformation of the publishing system, as well as fears, e.g. that transformation agreements unduly disadvantage other publishers. To investigate such questions, the publication output of Germany, Austria and Switzerland was examined. No negative effects of transformative agreements were found, but the hopes for substantial transformation steps have not been fulfilled so far either.

\section{Keywords}

Transformationsvertrag; Open Access; Gold Open Access; Hybrid Open Access; DEAL; ESAC; DACH-Länder; Deutschland; Österreich; Schweiz

\section{Einleitung $\underline{1}$}

Klassischerweise haben wissenschaftliche Bibliotheken Subskriptionsverträge für elektronische Zeitschriften abgeschlossen, die den Angehörigen der Einrichtungen den Zugriff auf Zeitschriften erlauben, welche ansonsten hinter einer Paywall sind („closed access“). Im Unterschied dazu werden für Gold-Open-Access-Zeitschriften keine institutionellen Verträge benötigt, weil hier der Zugriff frei möglich ist. Allerdings werden zur Abwicklung der oftmals anfallenden Publikationsgebühren (Article Processing Charges, APC) sowie zu deren Rabattierung in vielen Fällen doch Verträge zwischen Bibliotheken und Verlagen geschlossen. Zwischen diesen beiden Polen stehen Transformationsverträge. Sie beziehen sich auf Subskriptionszeitschriften, 
enthalten aber eine „Open-Access-Komponente“ und haben das Ziel, die Subskriptionszeitschriften in Open-Access-Zeitschriften zu transformieren (Shieber, 2014). Auch wenn eine kanonische Definition von Transformationsverträgen nicht existiert und in der Praxis ganz verschiedene Modelle umgesetzt sind, so haben sie doch zumindest die Gemeinsamkeit, dass neben dem Zugriff auf die Zeitschrifteninhalte („Read“) auch eine Open-Access-Publikationsmöglichkeit („Publish“) für die Angehörigen der betreffenden Einrichtung enthalten ist, ohne dass die Gefahr des „Double Dipping“ (Mittermaier,_2015). besteht.

Je nach Vertrag werden verschiedene Fragen unterschiedlich beantwortet:

- Sind alle Zeitschriften des Verlags berücksichtigt?

- Orientiert sich die Bezahllogik am (historischen) Subskriptionsvolumen oder am Publikationsaufkommen?

- Ist der Vertrag (ungefähr) kostenneutral oder steigen im Vergleich zu einem Subskriptionsvertrag die Ausgaben deutlich?

- Ist die Anzahl möglicher Open Access-Publikationen gedeckelt?

- Enthält der Vertrag Aussagen zum Flipping der Zeitschriften in den Open Access?

- Ist der Vertragstext selbst open?

Die ESAC Community hat Guidelines $\underline{2}$ veröffentlicht, welche die anzustrebenden Konditionen beschreiben. Diese werden in der Praxis - so auch in den im ESAC Registry $\underline{3}$ verzeichneten Transformationsverträgen - in unterschiedlichem Mass erfüllt. Mit Transformationsverträgen verbinden sich Hoffnungen und Befürchtungen, je zwei seien exemplarisch genannt:

1. Die Hoffnung, dass aufgrund von Transformationsverträgen der Open-Access-Anteil signifikant steigt.

2. Die Hoffnung, dass Transformationsverträge das Flipping von Subskriptionszeitschriften zu Gold-Open-Access-Zeitschriften bewirken.

3. Die Befürchtung, dass Transformationsverträge eine Gefahr für Gold-Open-AccessVerlage darstellen.

4. Die Befürchtung, dass Transformationsverträge mit grossen Verlagen eine Gefahr für kleine und mittlere Verlage darstellen.

Im Weiteren werden diese Hoffnungen und Befürchtungen datenbasierten „Realitätschecks“ unterzogen. Verwendet wird hierfür der vom Forschungszentrum Jülich entwickelte und betriebene „Open Access Monitor“ (Mittermaier et al.,_2018; 
Mittermaier,_2021a); für die Auswertungen wurden Daten von Unpaywall $\underline{4}$, gemappt mit Affiliationen des Web of Science $\underline{5}$ für Deutschland, Österreich und die Schweiz verwendet (DACH-Länder). Alle Auswertungen sind als Supplement Open Access verfügbar (Mittermaier, 2021b).

\section{Entwicklung des Open-Access-Anteils}

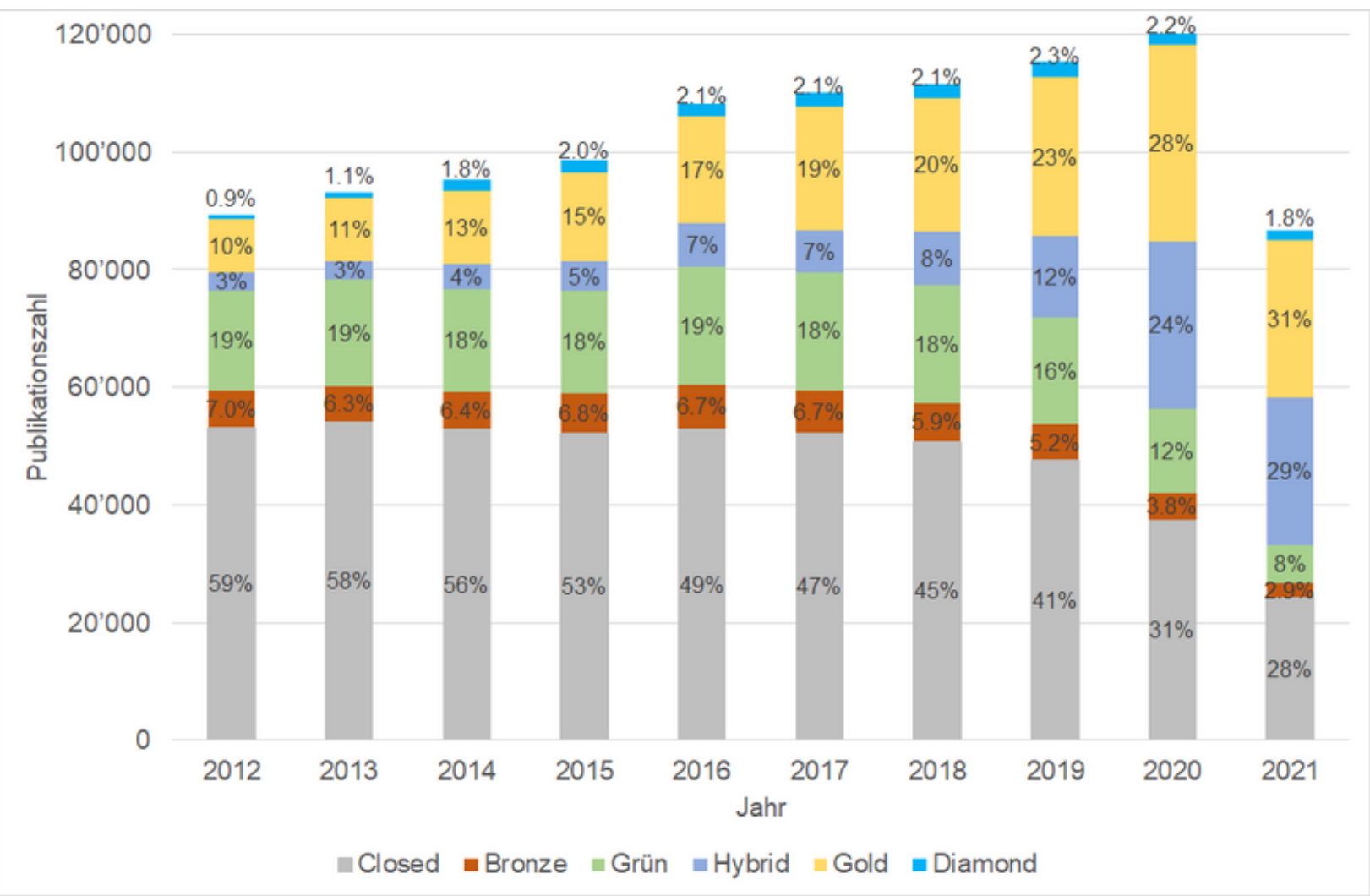

Abb. 1: Open-Access-Anteile 2012-2021 in den DACH-Ländern (Unpaywall/Web of Science, nur Corresponding Author-Publikationen, Stand 17.10.2021)

In den letzten zehn Jahren ist der Anteil der Artikel von Autor:innen aus den DACHLändern, die sich hinter einer Paywall befinden, von 59 \% um über die Hälfte auf nunmehr $\underline{6} 28 \%$ gesunken (Abbildung 1). Nimmt man zusätzlich die Nachnutzbarkeit in den Blick, welche bei Bronze Open Access gleichfalls nicht gegeben ist, so ist der Anteil der Artikel, die der Budapest-Definition „free and unrestricted online availability" (Chan et al.,2002). entsprechen, von 33 \% auf 69 \% gestiegen. Den Hauptanteil machen dabei Gold-Open-Access-Artikel (Anstieg von $10 \%$ auf $31 \%$ ) sowie Hybrid-Artikel aus. Ihr Anteil stieg noch deutlich stärker von $3 \%$ auf $29 \%$ und hat sich damit innerhalb einer Dekade etwa verzehnfacht. Beim Vergleich der Länder untereinander sind sich Deutschland und die Schweiz ziemlich ähnlich, Österreich hat 
dagegen durchweg noch höhere Hybridanteile. Diese stiegen von 9 \% in 2012 auf $37 \%$ in 2021. Detaillierte Daten sind als Supplement unter https://data.fzjuelich.de/api/access/datafile/4492 verfügbar. Insgesamt kann man konstatieren, dass der Open-Access-Anteil in der Tat deutlich gestiegen ist und dass dies zu einem guten Teil den Hybrid-Artikeln zu verdanken ist. Diese können aus Transformationsverträgen stammen, müssen es aber nicht, denn seit langem können Autor:innen eigeninitiativ Hybrid-Open-Access-Gebühren bezahlen. Wie weiter unten gezeigt wird, ist der markante Anstieg in den letzten Jahren (zum Beispiel Deutschland 2018: 7 \%, 2021: 28 \%) jedoch eindeutig auf Transformationsverträge zurückzuführen.

\section{Flipping von Subskriptionszeitschriften}

Insbesondere bei grossen Verlagen sind Zeitschriftenportfolios nicht statisch:

Zeitschriften stellen das Erscheinen ein, andere werden gegründet (und zwar sowohl im Subskriptions- als auch im Open-Access-Modell-그). Ebenfalls in beiden Geschäftsmodellen werden Zeitschriften von anderen Verlagen übernommen, weitere Zeitschriften werden an andere Verlage abgegeben. Und schliesslich können Zeitschriften das Geschäftsmodell ändern, indem sie vom Subskriptionsmodell zum Open-Access-Modell flippen oder auch in einem „reverse flip“ den umgekehrten Weg gehen. Angesichts dieser Bewegungen ist es wenig hilfreich, der Spur einzelner Zeitschriften zu folgen, jedoch sollte es beim Blick auf das gesamte Verlagsportfolio bemerkbar sein, wenn Transformationsverträge „to a swift and efficient transition of subscription journal portfolios to open access“ (DEAL Operations, 2021). beitragen. 


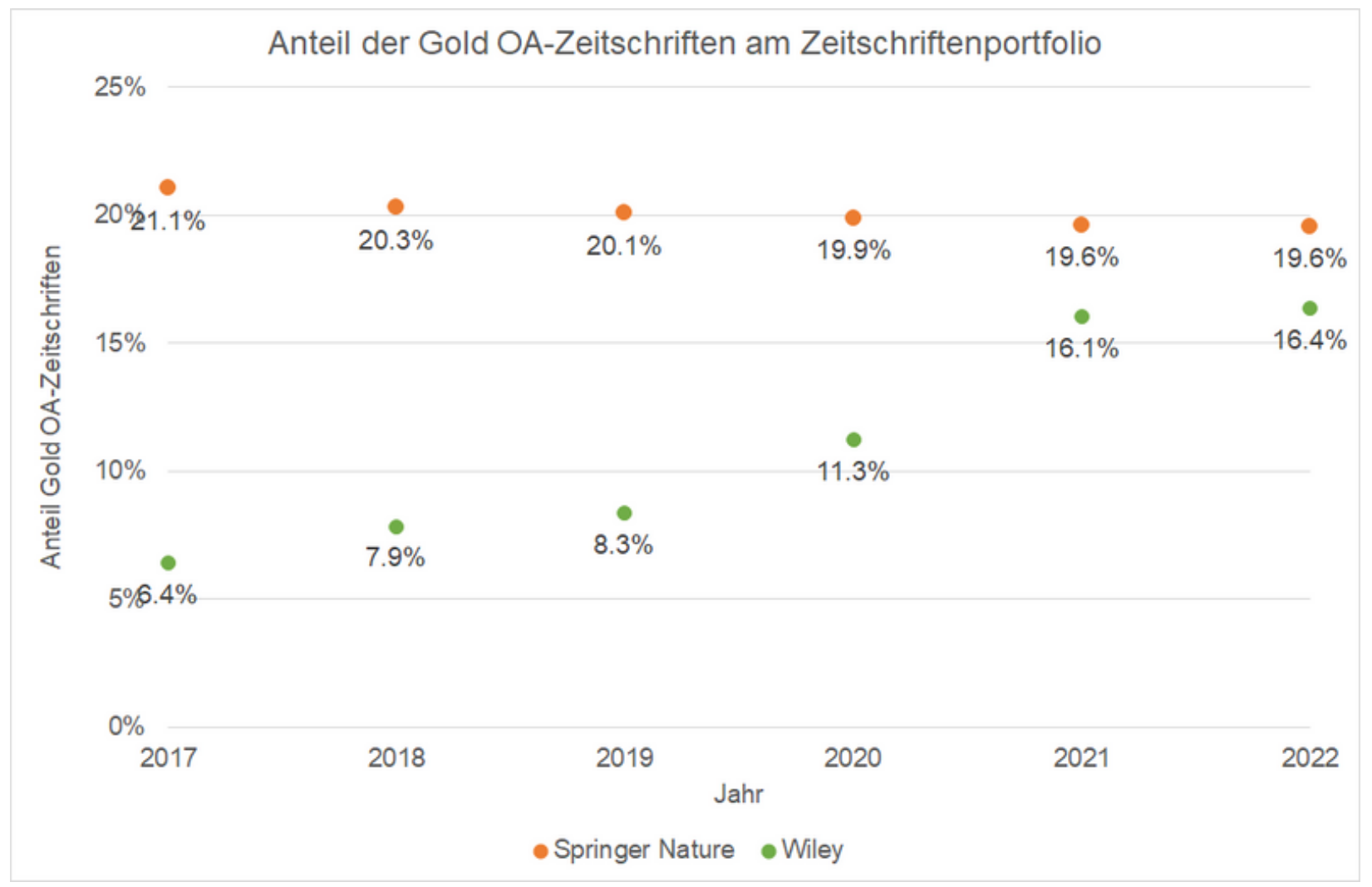

Abb. 2: Anteil der Gold Open Access-Zeitschriften an den Verlagsportfolios von Springer Nature ${ }^{8}$ und Wiley $\underline{9}$

Abbildung 2 (Daten unter https://data.fz-juelich.de/api/access/datafile/4490) zeigt die Entwicklung des Anteils der Open-Access-Zeitschriften bei zwei exemplarisch ausgewählten Verlagen. Springer Nature (damals Springer) hat 2008 den seinerzeit grössten Open-Access-Verlag BiomedCentral gekauft. In der Folge hat Springer Nature mit rund $20 \%$ einen relativ grossen Anteil Open-Access-Zeitschriften. Allerdings steigt dieser seit 2017 nicht an, sondern sinkt sogar leicht - wohl auch ein Ausdruck des Umstands, dass gemäss einer Untersuchung (Matthias, Jahn, \& Laakso, 2019). Springer Nature der Verlag mit den meisten „reverse flips“ ist, gefolgt von Elsevier und Taylor \& Francis. Wiley hat dagegen erst 2011 die ersten Gold-Open-AccessZeitschriften gegründet und seitdem das Portfolio kontinuierlich ausgebaut. Im Zeitraum 2015-2022 stieg der Anteil der Gold-Open-Access-Zeitschriften von 6,4 \% auf $16,4 \%$. Er liegt damit zwar immer noch niedriger als der von Springer Nature, allerdings ist hier eine Tendenz zu Open Access klar erkennbar. Dies zeigt sich in der Entwicklung der Quote ebenso wie in den einzelnen Aktivitäten: Neun der zehn für 2022 geplanten Neugründungen von Zeitschriften sind Open-Access-Titel. Weitere neun Titel flippen von Closed Access zu Open Access. Insofern findet bei Wiley 
tatsächlich ein Flipping statt, wenn auch nicht in dem Ausmass wie es der Anstieg des Anteils an Open-Access-Zeitschriften vermuten liesse. Bei Springer Nature stagniert dagegen der Anteil an Open-Access-Zeitschriften beziehungsweise sinkt leicht; von einem Umstieg in das Open-Access-Geschäftsmodell kann daher nicht die Rede sein. Als Fazit ist festzustellen, dass Transformationsverträge (auch die DEAL-Verträge) keine Selbstläufer für die Umstellung des Publikationswesens auf Open Access sind, sondern nur dann einen effektiven Beitrag leisten können, wenn es der faktischen Firmenpolitik entspricht.

\section{Transformationsverträge in der Kritik}

An Transformationsverträgen wird zum einen kritisiert, dass sie angeblich die Arbeit von Open-Access-Verlagen behindern (.(Eysenbach, 2020); OASPA Fully_OA Journal Publishers Interest Group, 2021) und zum anderen, dass sie, wenn sie mit grossen Verlagen abgeschlossen werden, angeblich die Marktanteile kleiner und mittlerer Verlage schmälern (Haucap,_Moshgbar, \& Schmal,_2021). „Die Marktchancen kleinerer Verlage sinken drastisch, da ihre Open-Access-Projekte nicht mehr wettbewerbsfähig sind“, klagt Christian Sprang, der Justiziar des Börsenvereins des Deutschen Buchhandels (Buchreport, 2019).

Um diese Vorwürfe faktenbasiert zu untersuchen, wurde die Entwicklung der Publikationen aus Deutschland, Österreich und der Schweiz auf Verlagsebene in den Jahren 2015 bis 2020 untersucht. Die Verlage wurden dabei länderspezifisch und zeitabhängig unterschieden in Gold OA-Verlage (ausschliesslich Gold-Open-AccessZeitschriften), Transformationsverlage (im betreffenden Land und Jahr überwiegend mit Transformationsverträgen am Markt) und Subskriptionsverlage (übrige Verlage). Das Merkmal „Open-Access-Verlag“ kann aus dem Open Access Monitor direkt abgelesen werden. Ob ein Verlag ein Transformationsverlag in diesem Sinne ist, wurde anhand der Einträge im ESAC Registry beurteilt. Zwangsläufig mussten hierbei „Schwarz/Weiss“-Entscheidungen getroffen werden. Als „Transformationsverlag“ galten nur Verlage, wenn der überwiegende Anteil ihres Portfolios beinhaltet ist (Gegenbeispiel: Thieme in Deutschland mit nur einer Zeitschrift im Vertrag) und wenn es sich um einen landesweiten Vertrag handelt (Gegenbeispiel: die Verträge des CERN und der Max Planck Digital Library, die nur ca. 5\% des nationalen Publikationsvolumens betreffen). Ein Indiz dabei war auch der im ESAC Registry angegebene Schätzwert für die Zahl der Publikationen, welcher mit den Realzahlen abgeglichen werden konnte. Anhand dieser Kriterien wurden die in Tabelle 1 
verzeichneten Verträge berücksichtigt (vollständige Übersicht in https://data.fzjuelich.de/api/access/datafile/4491).

\begin{tabular}{|l|l|c|}
\hline Land & Verlag & Vertragsjahre \\
\hline Deutschland & Cambridge University Press & $2019-2021$ \\
\hline Deutschland & IOP Publishing & $2019-2021$ \\
\hline Deutschland & Royal Society of Chemistry & $2018-2020$ \\
\hline Deutschland & Springer Nature & $2020-2022$ \\
\hline Deutschland & Wiley & $2019-2021$ \\
\hline Österreich & American Chemical Society & $2020-2022$ \\
\hline Österreich & Cambridge University Press & $2020-2022$ \\
\hline Österreich & Emerald & $2017-2020$ \\
\hline Österreich & IOP Publishing & $2014-2022$ \\
\hline Österreich & IWA Publishing & $2019-2021$ \\
\hline Österreich & Royal Society of Chemistry & $2017-2020$ \\
\hline Österreich & Sage & $2016-2021$ \\
\hline Österreich & Springer Nature & $2016-2021$ \\
\hline Österreich & Taylor \& Francis & $2014-2022$ \\
\hline Österreich & Wiley & $2018-2023$ \\
\hline Schweiz & Cambridge University Press & $2019-2020$ \\
\hline Schweiz & Elsevier & $2020-2022$ \\
\hline Schweiz & Karger & $2020-2023$ \\
\hline
\end{tabular}

Tab. 1: Nationale Transformationsverträge aus dem ESAC Registry mit Wirkung für die Jahre 2015-2020 (Stand 17.08.2021)

Die aus dem Open Access Monitor gewonnenen und nach diesen Kriterien zugeordneten Publikationszahlen sind unter https://data.fzjuelich.de/api/access/datafile/4493 als Supplement publiziert. Tabelle 2 enthält beispielhaft für Österreich die Zuordnung der fünf grössten Verlage (die Grösse wurde nach dem Publikationsaufkommen 2015-2020 bemessen). 


\begin{tabular}{|l|l|r|r|r|r|r|r|}
\hline Verlag & Modell & $\mathbf{2 0 1 5}$ & $\mathbf{2 0 1 6}$ & $\mathbf{2 0 1 7}$ & $\mathbf{2 0 1 8}$ & $\mathbf{2 0 1 9}$ & $\mathbf{2 0 2 0}$ \\
\hline Elsevier & Subskription & 2.274 & 2.511 & 2.361 & 2.406 & 2.545 & 2.488 \\
\hline Springer Nature & gemischt & 1.901 & 2.226 & 2.261 & 2.384 & 2.552 & 2.732 \\
\hline Wiley & gemischt & 1.119 & 1.129 & 1.142 & 1.138 & 1.139 & 1.131 \\
\hline MDPI & Gold & 125 & 177 & 251 & 471 & 847 & 1.342 \\
\hline Taylor \& Francis & Transformation & 413 & 464 & 480 & 498 & 508 & 521 \\
\hline
\end{tabular}

Tab. 2: Publikationen aus Österreich (Auszug). Mit Elsevier (weiss) gab es 2015-2020 keinen Transformationsvertrag. Mit Springer Nature und Wiley (zum Teil blau) gab es seit 2016 beziehungsweise 2018 nationale Transformationsverträge, vorher nur Subskriptionsverträge. MDPI (gold) ist ein Gold Open Access-Verlag. Mit Taylor \& Francis (blau) gab es durchgehend Transformationsverträge. $\underline{10}$

Wenn es ausser diesen Verlagen keine anderen gäbe, dann sähen die Publikationszahlen in den einzelnen Geschäftsmodellen aus wie in Tabelle 3 dargestellt:

\begin{tabular}{|l|r|r|r|r|r|r|}
\hline & $\mathbf{2 0 1 5}$ & $\mathbf{2 0 1 6}$ & $\mathbf{2 0 1 7}$ & $\mathbf{2 0 1 8}$ & $\mathbf{2 0 1 9}$ & $\mathbf{2 0 2 0}$ \\
\hline Subskription & 5.294 & 3.640 & 3.503 & 2.406 & 2.545 & 2.488 \\
\hline Transformation & 413 & 2.690 & 2.741 & 4.020 & 4.199 & 4.384 \\
\hline Gold & 125 & 177 & 251 & 471 & 847 & 1.342 \\
\hline
\end{tabular}

Tab. 3: Publikationen in den drei Geschäftsmodellen aus Österreich unter ausschliesslicher Berücksichtigung der Verlage in Tabelle 2.

Für die nachfolgend dargestellten Entwicklungen der Anteile muss die Publikationszahl eines Jahres ins Verhältnis zum Vorjahr gesetzt werden. Dies ist bei gleichbleibenden Verhältnissen trivial, zum Beispiel erfuhr „Gold“ 2016 eine Steigerung von $\frac{177}{125}-1=42 \%$ gegenüber 2015. Bei „Transformation“ wäre die gleiche Rechnung $\frac{2690}{413}-1=551 \%$ jedoch völlig falsch, da die 2.226 Transformations-Artikel bei Springer Nature (Tabelle 2) aus dem Jahr 2016 ins Verhältnis zu den 1.901 Subskriptionsartikeln im Vorjahr gesetzt werden müssen, ebenso die TransformationsArtikel bei Wiley aus dem Jahr 2018 zu den Subskriptionsartikeln aus dem Jahr 2017. Daher mussten die jeweiligen Nenner in der Berechnung der Steigerungsraten so berechnet werden, als seien die Verhältnisse im Vorjahr (Nenner) genauso wie im betrachteten Jahr (Zähler). Für die fünf Verlage aus Tabelle 2 ergibt dies die Werte in Tabelle 4 und mithin die Steigerungsraten/Rückgänge entsprechend Tabelle 5, beispielsweise Transformation 2016: $\frac{2690}{2314}-1=16 \%$. 


\begin{tabular}{|l|r|r|r|r|r|}
\hline & $\mathbf{2 0 1 5}$ & $\mathbf{2 0 1 6}$ & $\mathbf{2 0 1 7}$ & $\mathbf{2 0 1 8}$ & $\mathbf{2 0 1 9}$ \\
\hline Subskription & 3.393 & 3.640 & 2.361 & 2.406 & 2.545 \\
\hline Transformation & 2.314 & 2.690 & 3.883 & 4.020 & 4.199 \\
\hline Gold & 125 & 177 & 251 & 471 & 847 \\
\hline
\end{tabular}

Tab. 4: Hypothetische Publikationszahl in den drei Geschäftsmodellen aus Österreich unter ausschliesslicher Berücksichtigung der Verlage in Tabelle 2, wenn im jeweiligen Jahr die Verhältnisse des Folgejahrs geherrscht hätten.

\begin{tabular}{|l|r|r|r|r|r|}
\hline & $\mathbf{2 0 1 6}$ & $\mathbf{2 0 1 7}$ & $\mathbf{2 0 1 8}$ & $\mathbf{2 0 1 9}$ & $\mathbf{2 0 2 0}$ \\
\hline Subskription & $7 \%$ & $-4 \%$ & $2 \%$ & $6 \%$ & $-2 \%$ \\
\hline Transformation & $16 \%$ & $2 \%$ & $4 \%$ & $4 \%$ & $4 \%$ \\
\hline Gold & $42 \%$ & $42 \%$ & $88 \%$ & $80 \%$ & $58 \%$ \\
\hline
\end{tabular}

Tab. 5: Änderung der Publikationszahl gegenüber dem Vorjahr in den drei Geschäftsmodellen aus Österreich unter ausschliesslicher Berücksichtigung der Verlage in Tabelle 2.

Nach diesen methodischen Vorbemerkungen werden nachfolgend die Entwicklungen der relativen Anteile der einzelnen Geschäftsmodelle in Österreich, Deutschland und der Schweiz sowie abschließend für die DACH-Länder gemeinsam dargestellt: 


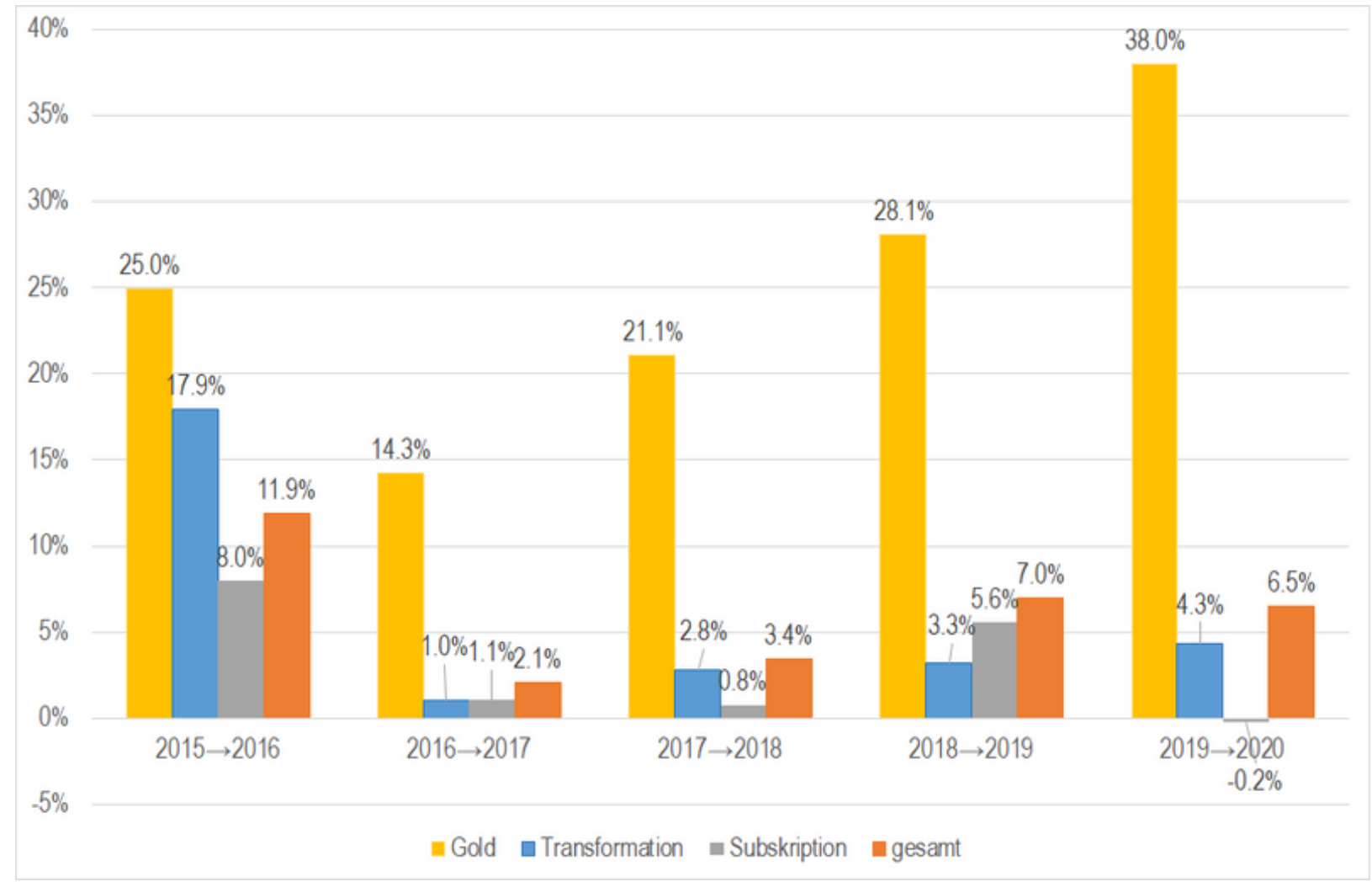

Abb. 3: Entwicklung der Publikationsanteile in den verschiedenen Geschäftsmodellen für Österreich

Österreich (Abbildung 3) hat die längste Tradition von Transformationsverträgen, manche reichen bis 2014 zurück. Der durchschnittliche jährliche Publikationszuwachs beträgt 6,1 \%. Transformationsverlage haben mit durchschnittlich 5,7 \% ein etwas niedrigeres Wachstum, Subskriptionsverlage wachsen mit durchschnittlich 3,0 \% nur halb so stark wie alle Verlage. Deutliche Gewinner sind die Gold-Open-Access-Verlage, deren jährlicher Zuwachs im Schnitt bei 25,0 \% liegt. Am Ende des Betrachtungszeitraums ist die Entwicklung besonders pointiert: Gold OA $\underline{11}$ wächst um 38,0 \%; Subskriptionsverlage verzeichnen dagegen mit -0,2 \% sogar einen kleinen Rückgang. 


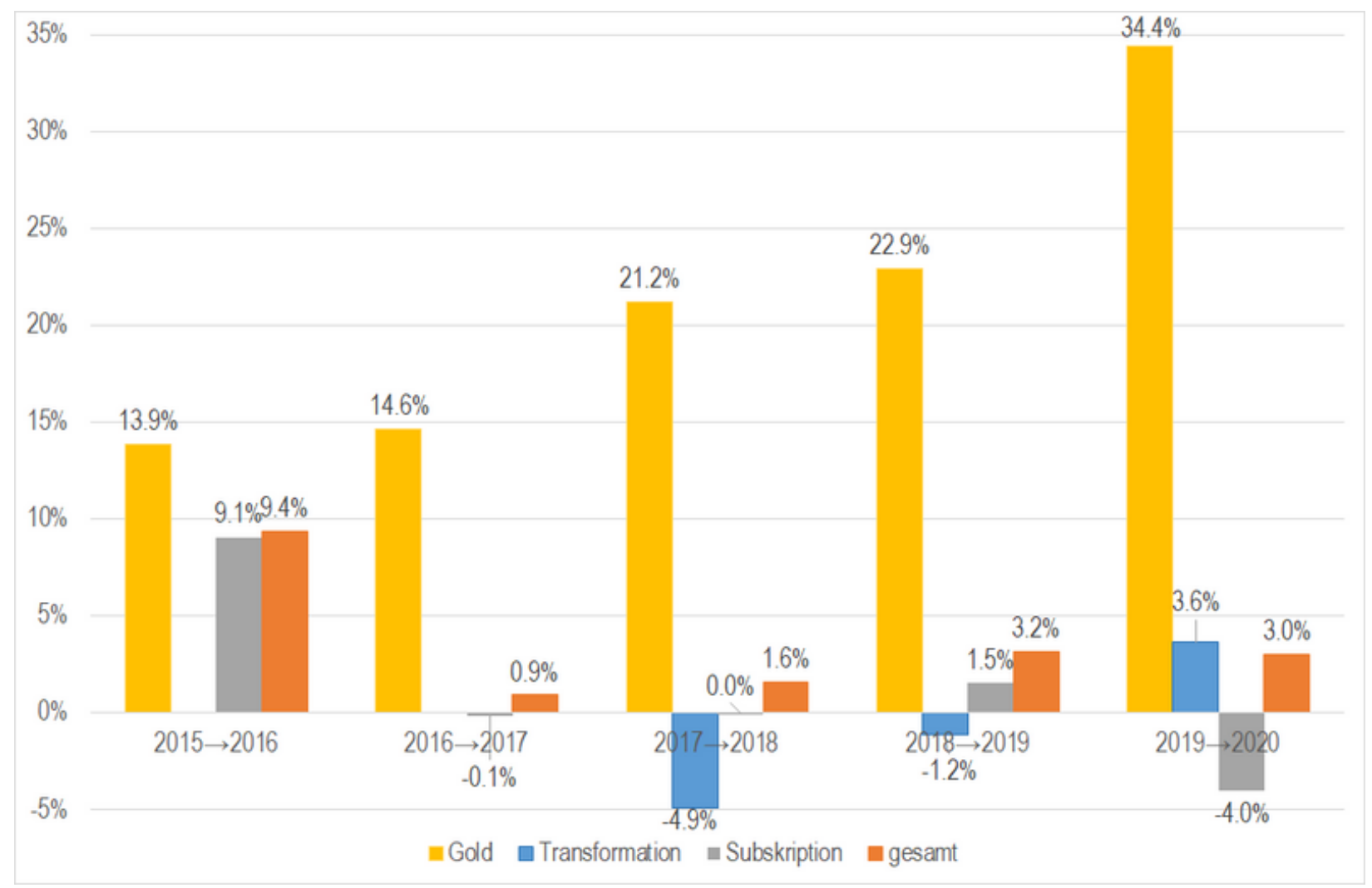

Abb. 4: Entwicklung der Publikationsanteile in den verschiedenen Geschäftsmodellen für Deutschland

In Deutschland (Abbildung 4) lag die durchschnittliche Zuwachsrate aller Publikationen im Gesamtzeitraum 2015-2020 bei 3,6 \% und damit niedriger als in Österreich. Auch hier ist der Zuwachs bei den Gold-Open-Access-Verlagen (21,2 \%) deutlich überdurchschnittlich und bei Subskriptionsverlagen $(1,2 \%)$ unterdurchschnittlich. Start des ersten nationalen Transformationsvertrags mit der Royal Society of Chemistry war 2018, inzwischen gibt es insgesamt fünf nationale Transformationsverträge $\underline{12}$. Transformationsverlage hatten zunächst Rückgänge zu verzeichnen und erst 2020 eine Steigerung, was insgesamt einen leichten Rückgang um 0,9\% ergibt. Dies erscheint auf den ersten Blick als Widerspruch zum Umstand, dass in absoluten Zahlen insgesamt ein Zuwachs um 870 Publikationen zu verzeichnen ist. $\underline{13}$ Die aktuellen Entwicklungen zeigen auch hier einen noch deutlich ausgeprägteren Kontrast: Während der Zuwachs bei Gold im letzten betrachteten Jahr noch zusätzlichen Auftrieb bekommen hat und nun bei 34,4 \% liegt, sanken dagegen Subskriptionsverlage um 4,0\%. 


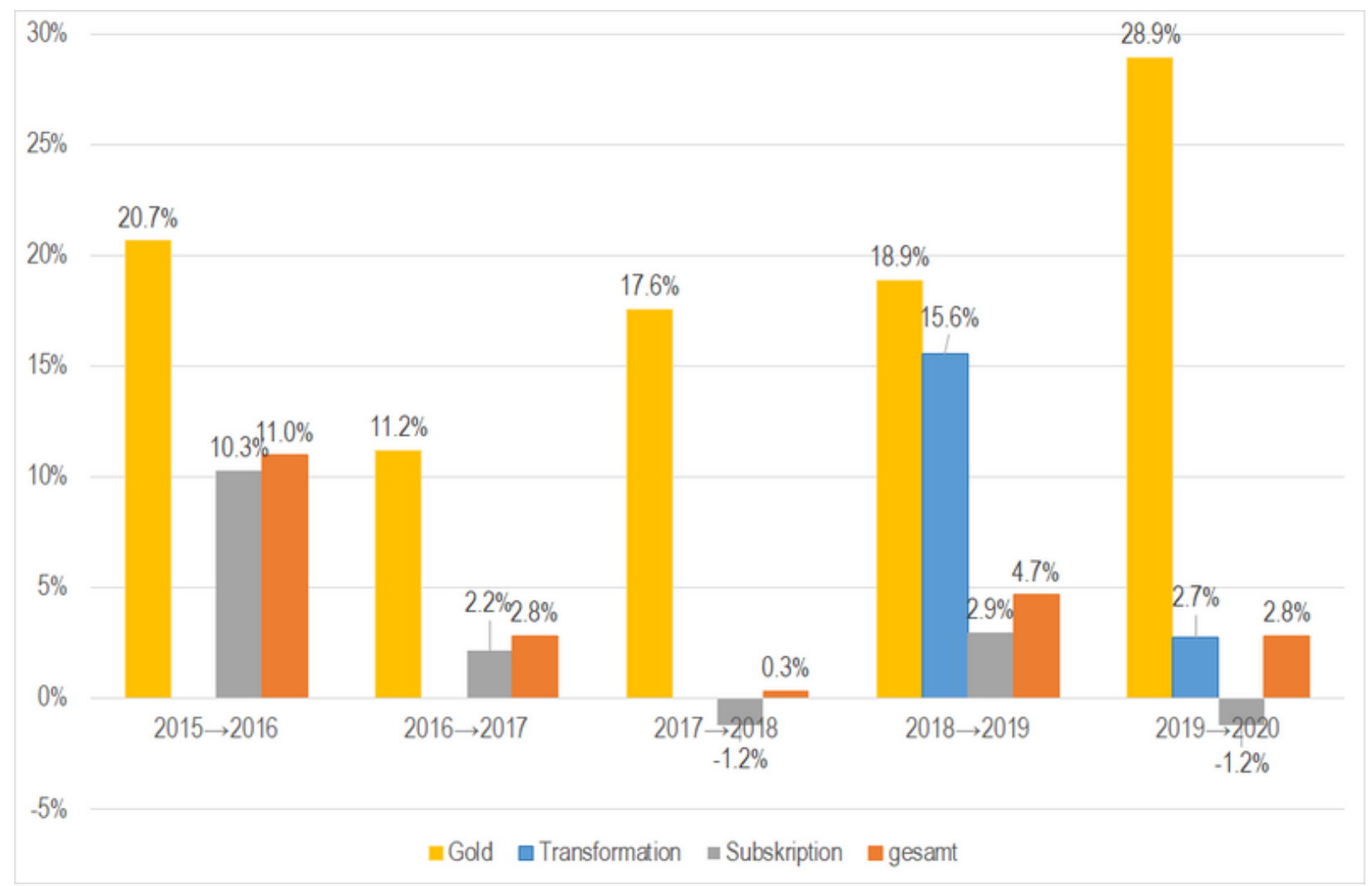

Abb. 5: Entwicklung der Publikationsanteile in den verschiedenen Geschäftsmodellen für die Schweiz

In der Schweiz (Abbildung 5) gibt es - ähnlich wie in Deutschland -

Transformationsverträge erst seit kurzem und noch nicht in grosser Zahl (ein Vertrag seit 2019, zwei Verträge seit 2020). Die durchschnittlichen Steigerungsraten aller Verlage $(4,3 \%)$ sowie der Subskriptionsverlage $(2,5 \%)$ liegen zwischen den Werten von Deutschland und Österreich. Die Steigerung bei den Gold OA-Verlagen ist mit durchschnittlich 19,3\% unter den drei Ländern am niedrigsten, aber immer noch weit über dem Durchschnitt aller Verlage. Die Steigerung bei den Transformationsverlagen liegt in der Schweiz mit 5,9 \% dagegen über den Werten der anderen Länder. Am Ende des Betrachtungszeitraum hat auch in der Schweiz die Steigerungsrate bei Gold OA den höchsten Wert aller Jahre, während die Subskriptionsverlage einen Rückgang verzeichnen. 


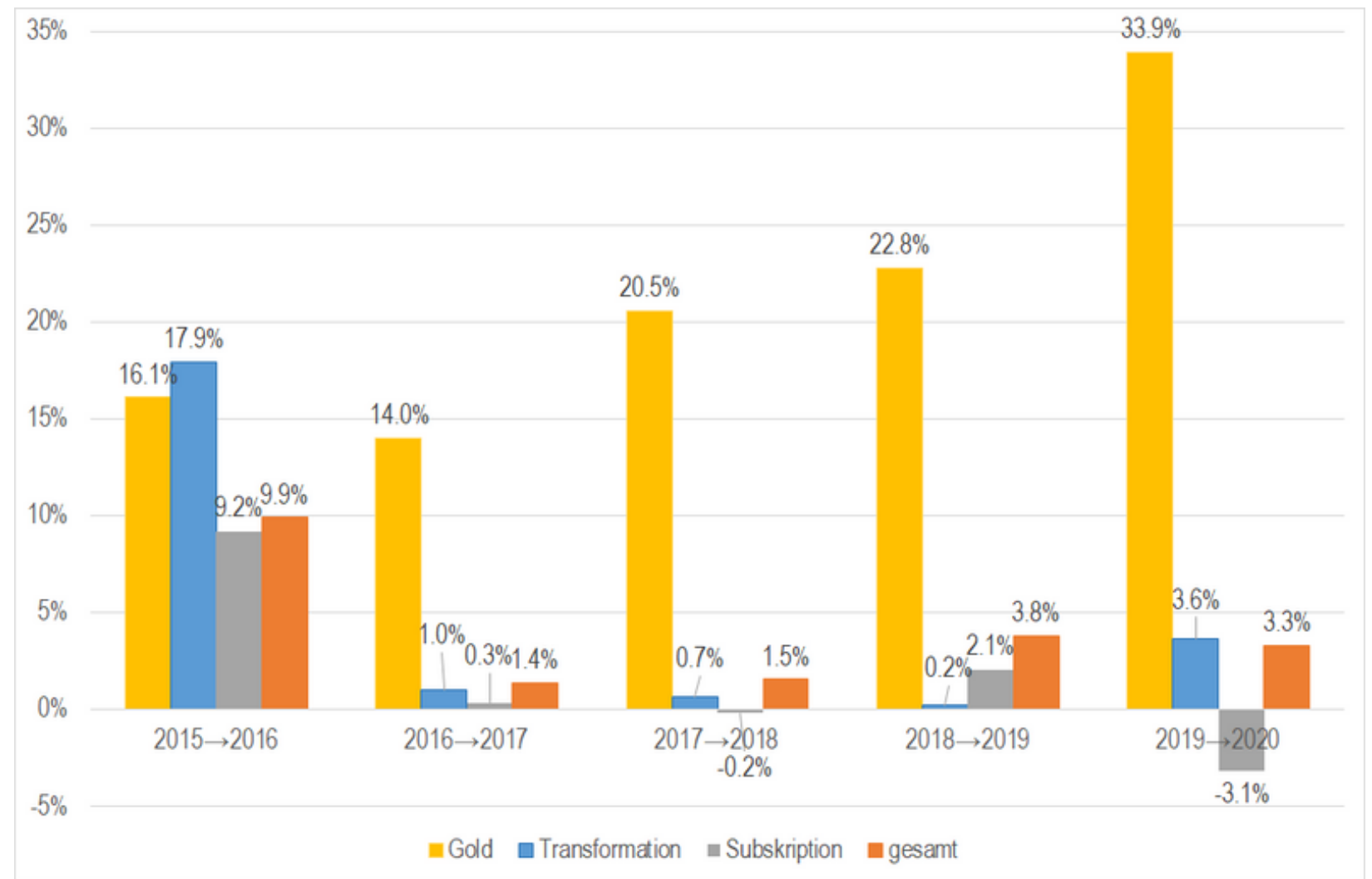

Abb. 6: Entwicklung der Publikationsanteile der DACH-Länder in den verschiedenen Geschäftsmodellen

In der Zusammenfassung der DACH-Länder (Abbildung 6) zeigt sich, dass alle Publikationsmodelle von 2015 nach 2016 grosse Zuwachsraten hatten, im Schnitt aller Verlage 9,9\%. In den Folgejahren haben die meisten Publikationsmodelle mit Ausnahme von Gold OA nur noch deutlich geringere Zuwachsraten bis hin zu Verlusten.

Über alle Jahre hinweg gibt es für alle Verlage zusammen („gesamt“) ein Artikelwachstum von durchschnittlich 3,9\%; von 2019 nach 2020 beträgt das Wachstum 3,3 \%. Ein "Corona-Effekt", also ein ungewöhnlicher Anstieg der Publikationszahlen im Jahr 2020 (Else,_2020), kann nicht festgestellt werden.

Die Werte für Transformationsverträge sind in manchen Jahren in der Betrachtung einzelner Länder nicht allzu belastbar, weil nur ein einziger Vertrag existiert. In der Zusammenfassung der drei Länder beruhen die Werte der Transformationsverlage dagegen auf mindestens 4 Verträgen mit 4 Verlagen (2015 2016) und auf bis zu 18 Verträgen mit 11 Verlagen (2019 2020). Die durchschnittliche Steigerungsrate ist mit $4,5 \%$ etwas grösser als im Durchschnitt aller Verlage, bei drei der fünf betrachteten Änderungsraten schneiden Transformationsverlage aber unterdurchschnittlich ab. 
Der Zuwachs bei Gold OA dominiert das Bild; er steigert sich von Jahr zu Jahr. Insgesamt haben Gold OA-Verlage durchschnittlich 21,3 \% Steigerung, jedoch gibt es deutliche Unterschiede zwischen den einzelnen Open-Access-Verlagen: Spitzenreiter sind MDPI und Frontiers mit durchschnittlich 56,7 \% beziehungsweise 24,1 \% jährlichem Wachstum; PLoS und Hindawi hatten dagegen Rückgänge von durchschnittlich 8,2 \% beziehungsweise 12,8\%.1ㅡㄹ Es ist somit nicht zutreffend, dass die Verhandlung mit grossen Hybrid-Verlagen „wettbewerbswidrig den Markt für

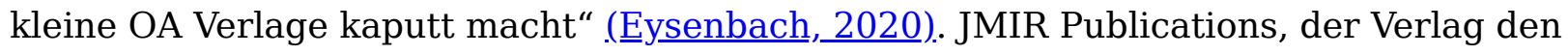
Gunther Eysenbach leitet, hatte in Deutschland ein durchschnittliches Wachstum von $51,0 \%$ (51,7 \% in den DACH-Ländern insgesamt).

Subskriptionsverlage haben seit 2016 insgesamt nur 1,6 \% Wachstum zu verzeichnen, verursacht insbesondere durch den Rückgang um 3,1 \% von 2019 auf 2020.

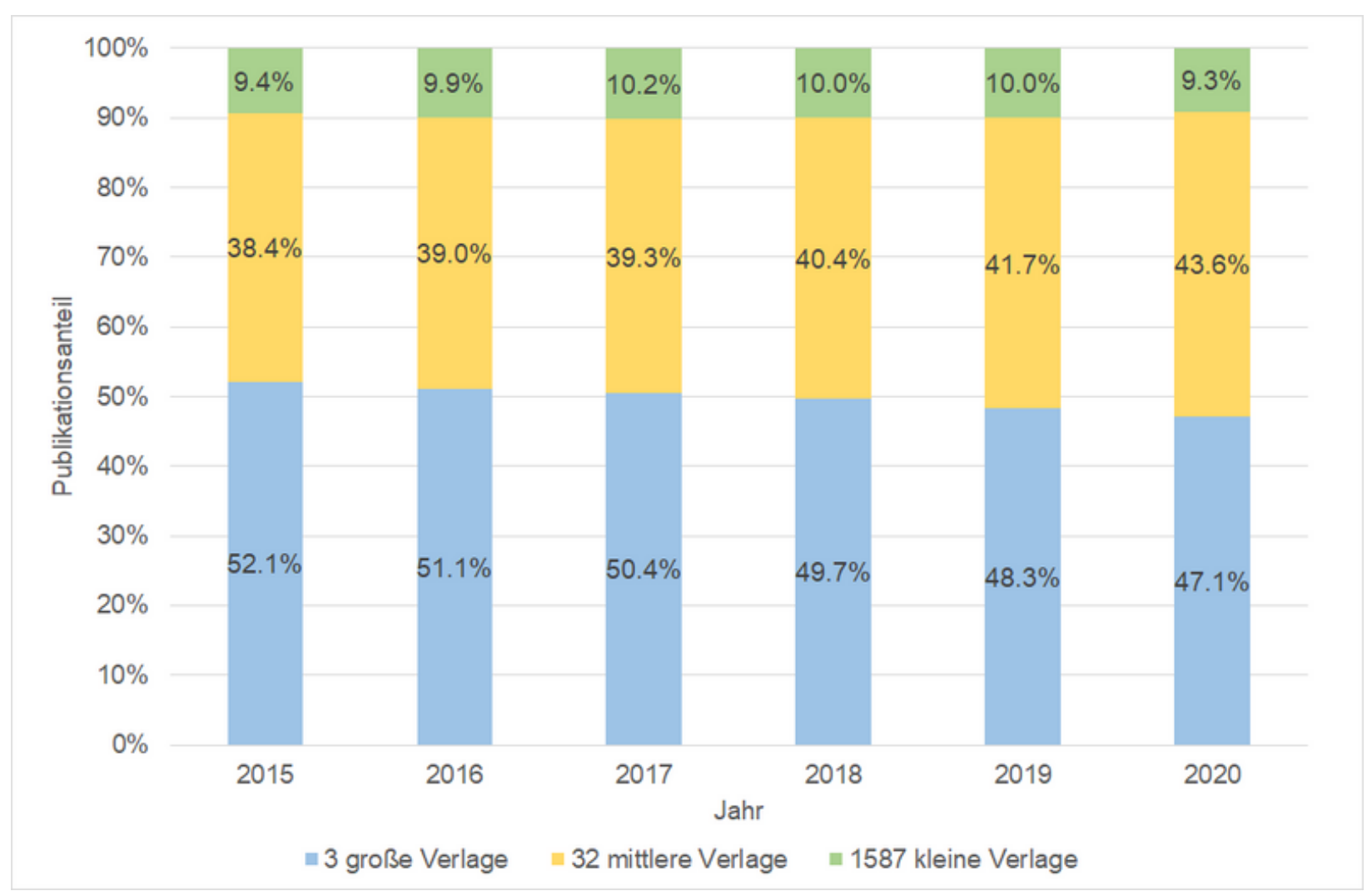

Abb. 7: Verteilung der Publikationsanteile der DACH-Länder auf grosse, mittlere und kleine Verlage. Grosse Verlage: > 10 \% Publikationsanteil. Kleine Verlage: < 0,25 \% Publikationsanteil.

Zur Frage der Auswirkung von Vertragsabschlüssen mit grossen Verlagen auf kleine und mittlere Verlage sind zunächst die drei Gruppen voneinander abzugrenzen. Die 
Abgrenzung der grossen Verlage Elsevier, Springer Nature und Wiley von den übrigen Verlagen ist einfach: Die drei Verlage haben zusammen rund 50 \% Publikationsanteil und einzeln jeweils mehr als $10 \%$. Der nächstgrössere Verlag MDPI hat in den DACHLändern 2015-2020 einen Anteil von 3,6 \%. Die Grenzziehung zwischen Wiley (10,8 \%) und MDPI liegt somit nahe. Nachfolgend gibt es keine markanten Sprünge mehr in den Publikationsanteilen; die Grenze zwischen mittleren und kleinen Verlagen wurde bei 0,20\% Anteil gezogen. Dieses Kriterium erfüllen weitere 32 Verlage, die zwischen $0,22 \%$ und 3,62 \% Publikationsanteil haben, zusammen rund $40 \%$. Zu diesen mittelgrossen Verlagen zählen unter anderen die schweizerischen Verlage Frontiers (Platz 9) und Karger (Platz 22) sowie die deutschen Verlage Thieme (Platz 11), De Gruyter (Platz 15), Copernicus (Platz 20) und Hogrefe (Platz 24). Die übrigen 1.587 Verlage, bei denen es 2015-2020 mindestens eine Publikation aus einem DACH-Land gab, werden zu den kleinen Verlagen gezählt. Abbildung 7 ist zu entnehmen, dass die grossen Verlage während des Betrachtungszeitraums insgesamt Anteile verloren haben (Rückgang von 52,1 \% auf 47,1 \%). Nutzniesser waren die mittelgrossen Verlage, deren Anteil von 38,4 \% auf 43,6 \% stieg. Der Anteil der kleinen Verlage ist am Anfang und am Ende des Betrachtungszeitraums praktisch unverändert. Hier wie auch bei den mittelgrossen Verlagen schwanken die Anteile im Lauf des Betrachtungszeitraums leicht; der Anteil der grossen Verlage geht dagegen kontinuierlich zurück. Die eingangs formulierte Befürchtung kann somit als widerlegt betrachtet werden, erst recht dann, wenn man wie der Börsenverein des Deutschen Buchhandels „die 'Quasi-Exklusivvereinbarungen’ mit den Grossverlagen Elsevier, Springer Nature und Wiley" (Buchreport, 2019). gegen alle anderen Verlage setzt letztere haben den Unkenrufen zum Trotz ihren Marktanteil von 47,9 \% auf 52,9 \% gesteigert.

\section{Zusammenfassung}

In diesem Artikel wurde die Entwicklung der Publikationszahlen im Rahmen nationaler Transformationsverträge in Deutschland, Österreich und der Schweiz in den Jahren 2015-2020 untersucht. Die Situation im Publikationswesen ist in vielerlei Hinsicht sehr volatil, unter anderem haben die DACH-Länder (mindestens) 17 neue Transformationsverträge mit einer Laufzeit ab 2021 abgeschlossen, was die Gesamtzahl der Verträge fast verdoppelt. Eine Neufassung der Studie im Jahr 2022, die dann auch das Jahr 2021 abdeckt, ist somit angezeigt.

Transformationsverträge eröffnen die Möglichkeit, in Subskriptionszeitschriften unter Bedingungen Open Access zu publizieren, die nicht einseitig vom Verlag diktiert sind 
und „Double Dipping“ ausschliessen. Eine positive Auswirkung von Transformationsverträgen ist der signifikante Anstieg des Anteils der Publikationen, die Open Access erscheinen, wobei der gegenwärtig beobachtete deutliche Anstieg der Open-Access-Anteile in noch grösserem Ausmass auf Publikationen in Gold-OpenAccess-Zeitschriften zurückzuführen ist.

Die Hoffnung, dass Transformationsverträge das Flipping von Subskriptionszeitschriften zu Gold-Open-Access-Zeitschriften bewirken, hat sich bislang nur in sehr bescheidenem Umfang verwirklicht. Gerade Springer Nature, der Verlag, der schon früh und in vielen Ländern Transformationsverträge abgeschlossen hat, scheint bislang keine Pläne zur Transformation von Subskriptionszeitschriften zu Open-Access-Zeitschriften zu verwirklichen. Angesichts dessen wird es interessant sein zu beobachten, wie die Verlage die behauptete „Plan S-Compliance“ erfüllen wollen. $\underline{15}$

Transformationsverträge stellen keine Gefahr für Gold-Open-Access-Verlage dar. Deren Wachstum ist insgesamt noch deutlich grösser als das Wachstum der Verlage mit Transformationsverträgen. Ebenso wenig stellen Transformationsverträge mit grossen Verlagen eine Gefahr für kleine und mittlere Verlage dar - dieser Vorwurf wurde dem Projekt DEAL in Deutschland mehrfach gemacht. Tatsächlich sinkt der Anteil der drei grossen Verlage am Publikationsaufkommen. Insgesamt ist die treibende Kraft der gegenwärtigen Änderungen im Publikationswesen der Wunsch der Autor:innen, Open Access zu publizieren - bevorzugt in Gold-Open-Access-Zeitschriften, daneben im Hybrid Open Access im Rahmen von Transformationsverträgen. Für Subskriptionszeitschriften stellen somit nicht Transformationsverträge, sondern das eigene Geschäftsmodell die grösste Gefahr dar. Transformationsverträge sind weder „Stairway to Heaven“ (Led Zeppelin) noch „Highway to Hell“ (AC/DC), sondern am ehesten als „The Long and Winding Road“ (Beatles) zu charakterisieren.

\section{Danksagung}

Herzlichen Dank an Susanne Aerni (Konsortium der Schweizer Hochschulbibliotheken) und Brigitte Kromp (Universität Wien) für die Überprüfung der Klassifikation von ESAC Registry-Eintragungen für Österreich beziehungsweise für die Schweiz.

\section{Footnotes}

1. Teile dieser Untersuchung wurden präsentiert bei der Internationalen Open Access Woche am 28.10.2021 https://juser.fz-juelich.de/record/902150 sowie bei 
OASPA 2021 Online Conference on Open Access Scholarly Publishing https://oaspa.org/wp-content/uploads/2021/09/Mittermaier.pdf (zuletzt geprüft: 24.10.2021). Bei der OASPA-Tagung wurden nicht Corresponding AuthorPublikationen zu Grunde gelegt, sondern alle Publikationen. $\triangleq$

2. https://esac-initiative.org/about/transformative-agreements/guidelines-fortransformative-agreements/ (zuletzt geprüft 24.10.2021)

3. https://esac-initiative.org/about/transformative-agreements/agreement-registryl (zuletzt geprüft 24.10.2021)

4. Unpaywall data feed: https://unpaywall.org/products/data-feed (zuletzt geprüft 24.10.2021)

5. Data sourced from Web of Science; a research information system provided by Clarivate Analytics (http://apps.webofknowledge.com).

6. Aus dem Jahr 2021 sind Publikationen bis 17. Oktober berücksichtigt. $\subseteq$

7. Dies ist offenbar der bei Elsevier beschrittene Weg; 2020 waren über $90 \%$ der neu gegründeten Zeitschriften Open-Access-Zeitschriften https://www.elsevier.com/open-access/open-access-journals (Zugriff am 24.10.2021). Viele der neuen Zeitschriften sind „Mirror Journals“, die mit gleichem Scope und oftmals gleichen Herausgeber:innen parallel zu einer bestehenden Subskriptionszeitschrift gegründet werden. Von Flipping kann hierbei natürlich nicht die Rede sein. $\subseteq$

8. https://www.springernature.com/gp/librarians/licensing/journals-catalog (Zugriff am 24.10.2021) enthält die Zeitschriftenlisten 2019-2022. Titellisten früherer Jahre hat der Autor gesammelt; sie sind durch Internetrecherchen zum Teil noch zugänglich. $\triangleq$

9. https://onlinelibrary.wiley.com/library-info/products/price-lists (Zugriff am 24.10.2021) enthält die Zeitschriftenliste 2022. Titellisten früherer Jahre hat der Autor gesammelt; sie sind durch Internetrecherchen zum Teil noch zugänglich. 10. Publikationen der American Geophysical Union (AGU) und der International Union of Crystallography (IUCr) sind Teil der Wiley-Verträge und deshalb in den Wiley-Zahlen enthalten; Hindawi-Publikationen sind nicht Teil der Wiley-Verträge und deshalb nicht in den Wiley-Zahlen enthalten. Neben diesen generell gültigen Aspekten gibt es für Deutschland noch eine weitere Besonderheit bei Wiley: Beim 
DEAL-Vertrag mit Wiley begann die Möglichkeit des Open-Access-Publizierens in Subskriptionszeitschriften am 01.07.2019 und somit unterjährig. Für die Zwecke dieser Untersuchung wird der Vertrag für das gesamte Jahr 2019 als Transformationsvertrag gewertet. $\_$

11. Es sei daran erinnert, dass unter „Gold Open Access“ in diesem Zusammenhang nur die Publikation bei reinen Open Access-Verlagen verstanden werden. Zusätzlich gibt es noch Publikationen in Gold Open Access-Zeitschriften anderer Verlage. $ヒ$

12. Zehn weitere Verträge starteten 2021, dies liegt aber außerhalb des Untersuchungszeitraums dieser Arbeit. $\Xi$

13. Die durchschnittliche Änderung ergibt sich als geometrisches Mittel \$ \sqrt[3] $\{95,1 \backslash \% * 98,8 \backslash \% * 103,6 \backslash \% \backslash\} \$-1$. Die absolute Änderung ist (-87-157+1114). Siehe

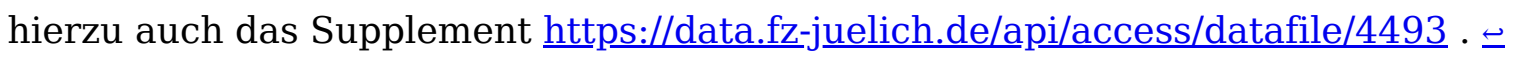

14. Weitere Auswertungen auf Verlagsebene enthält Mittermaier, B. (2021). Auswirkungen des „DEAL “ auf das Publikationsverhalten. Retrieved from http://hdl.handle.net/2128/27787.

15. Die in der Coalition S zusammengeschlossenen Forschungsförderer übernehmen Hybrid-Open-Access-Publikationsgebühren nur dann, wenn sich die Zeitschriften als „Transformative Journal“ qualifizieren (https://www.coalition-s.org/transformativejournals-faq/). In der Aufstellung https://www.coalition-s.org/plan-s-complianttransformative-journals/ hat Springer Nature über 1.700 Zeitschriften als „Transformative Journal“ registriert (https://www.springernature.com/gp/openresearch/transformative-journals) und Elsevier über 160 Zeitschriften (https://www.elsevier.com/ data/assets/pdf file/0004/1104808/ElsevierTransformativ eJournals.pdf). Wiley hat bislang sehr zurückhaltend auf Plan S reagiert (https://authorservices.wiley.com/statements/plan-s-implementation.html) (alle Seiten Stand 24.10.2021). $\subseteq$

\section{Citations}

1. Shieber, S. (2014). A true transitional open-access business model. The occasional pamphlet. Retrieved from http://blogs.harvard.edu/pamphlet/2014/03/28/a-truetransitional-open-access-business-model $/ \subseteq$

2. Mittermaier, B. (2015). Double Dipping beim Hybrid Open Access - Chimäre oder Realität? Informationspraxis, 1(1). https://doi.org/10.11588/ip.2015.1.18274 
3. Mittermaier, B., Barbers, I., Ecker, D., Lindstrot, B., Schmiedicke, H., \& Pollack, P. (2018). Der Open Access Monitor Deutschland. O-Bib, 5(4), 84-100.

https://doi.org/10.5282/o-bib/2018H4S84-100

4. Mittermaier, B. (2021). Die Rolle des Open Access Monitor Deutschland bei der Antragstellung im DFG-Förderprogramm „Open-Access-Publikationskosten“. $O$-Bib, 8(4). https://doi.org/10.5282/o-bib/5731

5. Mittermaier, B. (2021). Publikationsoutput DACH-Länder 2015-2020. Jülich DATA. https://doi.org/10.26165/JuELICH-DATA/3MPPCO

6. Chan, L., Cuplinskas, D., Eisen, M., Friend, F., Genova, Y., Guédon, J.-C., ...

Velterop, J. (2002). Budapest Open Access Initiative. Retrieved from

https://www.budapestopenaccessinitiative.org/read $\leftrightarrows$

7. DEAL Operations. (2021). The nationwide Projekt DEAL approach. Retrieved from https://deal-operations.de/en/here-is-the-deal/deal-approach $\triangleq$

8. Matthias, L., Jahn, N., \& Laakso, M. (2019). The two-way street of open access journal publishing: flip it and reverse it. Publications, 7(2), 23.

https://doi.org/10.3390/publications7020023

9. Eysenbach, G. (2020). Kommentar zu "Börsenverein legt»Deal«-

Kartellbeschwerde ein." Retrieved from https://www.buchreport.de/news/noch-

allianz-oder-schon-kartell/\#comment-41765

10. OASPA Fully OA Journal Publishers Interest Group. (2021). The Fully OA

agreement - an essential component of a diverse open access world. Guest Post. Retrieved from https://oaspa.org/guest-post-the-fully-oa-agreement-an-essentialcomponent-of-a-diverse-open-access-world/ $\leftrightarrows$

11. Haucap, J., Moshgbar, N., \& Schmal, W. B. (2021). The impact of the German "DEAL" on competition in the academic publishing market (DICE Discussion Paper, 360). Düsseldorf: Heinrich Heine University Düsseldorf, Düsseldorf Institute for Competition Economics (DICE). Retrieved from http://hdl.handle.net/10419/231802 12. Buchreport. (2019). Börsenverein legt»Deal«-Kartellbeschwerde ein. Retrieved from https://www.buchreport.de/news/noch-allianz-oder-schon-kartell/ 13. Else, H. (2020). How a torrent of COVID science changed research publishing in seven charts. Nature, 588(7839), 553. https://doi.org/10.1038/d41586-020-03564-y. 
027.7 Zeitschrift für Bibliothekskultur / Journal for Library Culture • Ausgabe 